\title{
Grenzbelastungen im Länderfinanzausgleich
}

\author{
Wolfgang Scherf (iD
}

Online publiziert: 5. August 2020

(C) Der/die Autor(en) 2020

Zusammenfassung Wachsende Steuereinnahmen eines Bundeslandes oder seiner Gemeinden führen zu geringeren Ausgleichsansprüchen im Länderfinanzausgleich. Dadurch entstehen hohe Grenzbelastungen des Landeshaushalts und des Gesamthaushalts von Land und Gemeinden. Ihre quantitative Bestimmung erfordert keine Simulation des gesamten Länderfinanzausgleichs. Der Autor entwickelt ein Modell, das eine exakte und transparente Ermittlung der Grenzbelastungen mithilfe der Einwohnerzahlen der Bundesländer und der Einwohnergewichte bei den Länder- und Gemeindesteuern ermöglicht. Triebfeder der Spitzenbelastungen sind die neuen Bundesergänzungszuweisungen für unterproportionale Gemeindesteuerkraft. Ihre Abschaffung empfiehlt sich als erstrangige Reformoption für eine anreizfreundlichere Gestaltung des Länderfinanzausgleichs.

Schlüsselwörter Umsatzsteuerverteilung · Finanzkraftausgleich · Bundesergänzungszuweisungen · Einwohnergewichte · Anreizeffekte

W. Scherf $(\varangle)$

Justus-Liebig-Universität Gießen, Gießen, Deutschland

E-Mail: wolfgang.scherf@wi.jlug.de 


\title{
Marginal burdens in the federal state fiscal equalization
}

\begin{abstract}
Growing tax revenues of a federal state or its municipalities lead to lower compensation claims in the federal state fiscal equalization. This creates high marginal burdens on the federal state budget and the overall budget of the states and municipalities. Their quantitative determination does not require a simulation of the entire federal state fiscal equalization. The author develops a model that enables an exact and transparent determination of marginal burdens using the population figures of federal states and the population weights for state and municipal taxes. The driving force behind the maximum burdens are the new supplementary federal grants for under-proportional municipal tax revenue. Their abolition is recommended as the primary reform option for a more incentive-friendly design of the federal state fiscal equalization.
\end{abstract}

Im deutschen Länderfinanzausgleich werden Mehreinnahmen eines Bundeslandes in hohem Maße durch geringere Ausgleichsansprüche abgeschöpft. Das senkt die Attraktivität eigener Anstrengungen zur Verbesserung der Finanzkraft und erzeugt Fehlanreize. Die Grenzbelastungen werden in der Regel durch Simulation des Länderfinanzausgleichs für den Fall zusätzlicher Einnahmen eines Landes aus einzelnen Steuern bestimmt. ${ }^{1}$ Dieser Weg ist relativ kompliziert und zeigt auch nicht transparent auf, wie die Grenzbelastungen zustande kommen. Der Beitrag präsentiert ein formales Modell, das mit wenigen Basisinformationen auskommt und dennoch eine exakte Bestimmung der Grenzbelastungen des aktuellen Systems und seiner Reformoptionen ermöglicht.

\section{Länderfinanzausgleich im Überblick}

Der Länderfinanzausgleich hat die Aufgabe, die Finanzkraftdifferenzen zwischen den Bundesländern zu reduzieren, damit auch die finanzschwächeren Länder ihre verfassungsmäßigen Aufgaben erfüllen können. ${ }^{2}$ Dieses Ziel soll mit drei verschiedenen Instrumenten erreicht werden.

- Auf der ersten Stufe des Länderfinanzausgleichs wird der Länderanteil an der Umsatzsteuer nach Einwohnern verteilt. Anschließend erfolgt ein Finanzkraftausgleich durch $\mathrm{Zu}$ - und Abschläge, die mit den Umsatzsteueranteilen verrechnet werden.

- Auf der zweiten Stufe erhalten die nach Finanzkraftausgleich immer noch relativ finanzschwachen Länder allgemeine Bundesergänzungszuweisungen, die den Abstand zum Durchschnitt weiter verkleinern.

\footnotetext{
${ }^{1}$ Simulationsrechnungen finden sich z. B. bei Büttner und Görbert (2016) und Burret et al. (2018).

2 Vgl. zur generellen Funktionsweise und zu den Wirkungen des Länderfinanzausgleichs Scherf (2020) und die dort angegebene Literatur. In diesem Beitrag geht es nur um die finanzkraftbezogenen Elemente des Systems. Davon unabhängige Regelungen für Sonderbedarfe bleiben außer Betracht.
} 
- Die dritte Stufe umfasst eine steuerkraftbezogene Sonderbedarfsregelung. Länder mit relativ steuerschwachen Gemeinden erhalten spezielle Bundesergänzungszuweisungen für ihre unterproportionale Gemeindesteuerkraft.

Der aktuelle Länderfinanzausgleich lässt sich durch ein Gleichungssystem darstellen. Alle ausgleichsrelevanten Größen werden dabei in Euro pro Einwohner gemessen. Das vereinfacht den Vergleich zwischen Ländern unterschiedlicher Größe und verdeutlicht die Ausgleichsfunktion des Systems, das im Kern auf eine Angleichung der Pro-Kopf-Finanzkraft der Länder gerichtet ist.

\section{Umsatzsteuerverteilung und Finanzkraftausgleich}

Die Steuereinnahmen der Länder und ihrer Gemeinden bestimmen die Finanzkraft der Länder vor Finanzausgleich. ${ }^{3}$ Sie werden zu einer Finanzkraftmesszahl aggregiert, die in Relation zu einer Ausgleichsmesszahl die relative Finanzkraft eines Landes abbildet.

Bezogen auf die tatsächlichen Einwohner ergeben die „Steuern der Länder im Finanzkraftausgleich“ die Steuerkraftmesszahl eines Landes $\left(\mathrm{SMZ}_{\mathrm{L}}\right)$. Daneben wird ein bestimmter Anteil $(\mathrm{g}=75 \%)$ von der Steuerkraftmesszahl der Gemeinden pro Einwohner $\left(\mathrm{SMZ}_{\mathrm{G}}\right)$ als Finanzkraft des Landes veranschlagt. Zusammen mit der Steuerkraftmesszahl des Landes resultiert daraus die Finanzkraftmesszahl eines Landes $\left(\mathrm{FMZ}_{\mathrm{L}}\right)$ :

$$
\mathrm{FMZ}_{\mathrm{L}}=\mathrm{SMZ}_{\mathrm{L}}+\mathrm{gSMZ}
$$

Der Länderfinanzausgleich orientiert sich an der Differenz zwischen der Ausgleichsmesszahl $\left(\mathrm{AMZ}_{\mathrm{L}}\right)$ und der Finanzkraftmesszahl eines Landes. Im Grundmodell des Finanzkraftausgleichs dient die durchschnittliche Finanzkraft pro Einwohner als Ausgleichsmesszahl. Im Länderfinanzausgleich werden jedoch die Einwohner einiger Länder nicht mit dem Faktor 1, sondern teils höher gewichtet. Die Einwohnergewichte unterscheiden sich zudem bei den Länder- und Gemeindesteuern.

Tab. 1 enthält die erforderlichen Basisdaten für die Ermittlung der Ausgleichsmesszahlen. In der Tabelle sind die Länder nach Flächenländern und Stadtstaaten gruppiert und innerhalb der Gruppen nach der Einwohnerzahl absteigend sortiert.

Aus der Einwohnerzahl eines Landes (E) ergibt sich in Relation zur Summe der Einwohner aller Länder ( $\sum E$ ) zunächst der Einwohneranteil eines Landes (e). Die gewichteten Einwohner werden nun getrennt nach Länder- und Gemeindesteuern $\left(E_{L}\right.$ bzw. $\left.E_{G}\right)$ ermittelt. Sie errechnen sich aus der tatsächlichen Einwohnerzahl mul-

\footnotetext{
3 Die Steuern der Länder betragen 2020 ca. 304,2 Mrd. €. Sie umfassen Gemeinschaftsteueranteile (Lohnsteuer: $217,4 \mathrm{Mrd} . €$, veranlagte Einkommensteuer: $62,3 \mathrm{Mrd}$. $€$, Abgeltungsteuer: $5 \mathrm{Mrd}$. $€$, nicht veranlagte Steuern vom Ertrag: 23,2 Mrd. €, Körperschaftsteuer: 32,4 Mrd. €), Gewerbesteuerumlage: $2,9 \mathrm{Mrd}$. $€$, Ländersteuern: $34,3 \mathrm{Mrd}$. $€$ (davon Erbschaftsteuer: 6,9 Mrd. $€$, Grunderwerbsteuer: $15,2 \mathrm{Mrd}$. $€$, Kraftfahrzeugsteuerkompensationsbeträge: $9 \mathrm{Mrd}$ €), Umsatzsteueranteile: $118 \mathrm{Mrd}$. $€$ und $33 \%$ der Förderabgabe. Hinzugerechnet werden $75 \%$ der Gemeindesteuern: 87,7 Mrd. $€$ (Einkommen- und Umsatzsteueranteile, Gewerbesteuer abzüglich Umlage, Grundsteuern).
} 


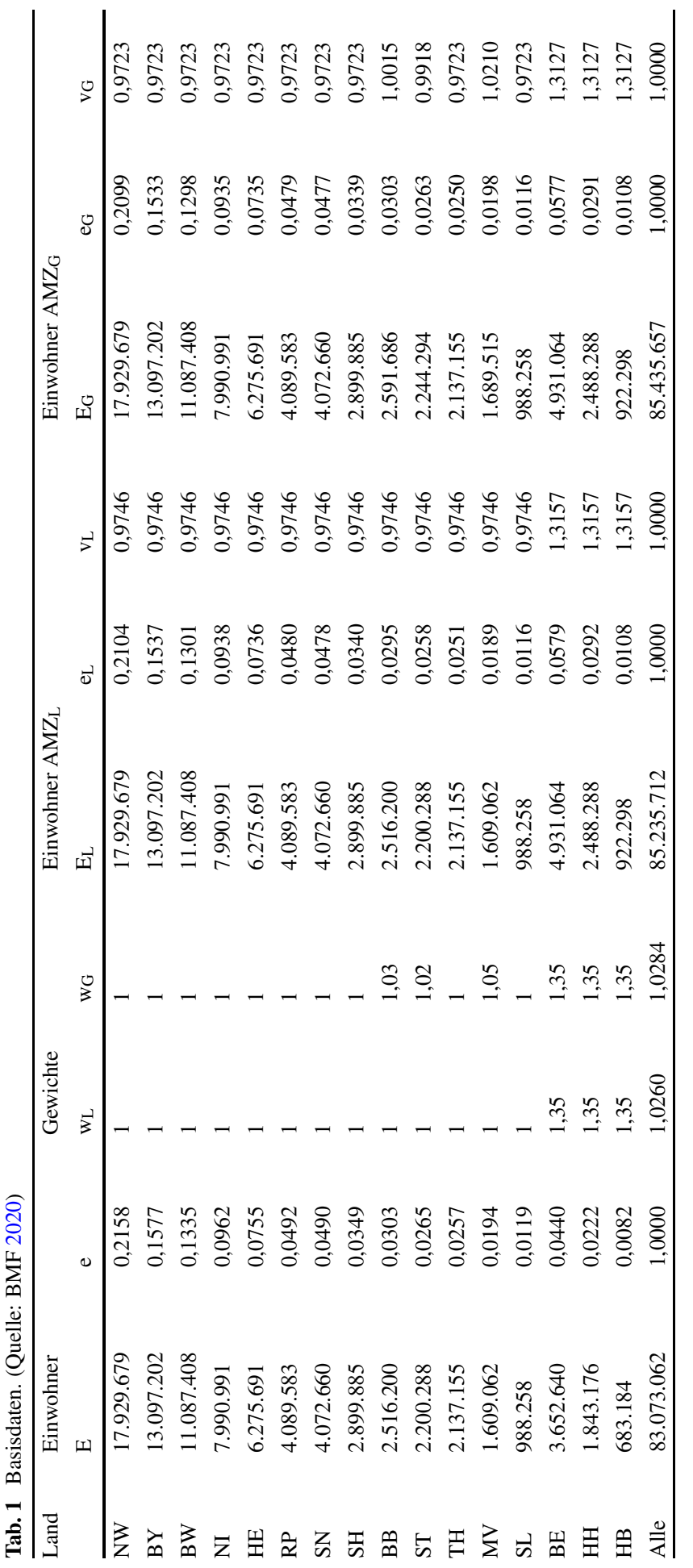


tipliziert mit den jeweiligen Einwohnergewichten $\left(\mathrm{w}_{\mathrm{L}}\right.$ bzw. $\left.\mathrm{w}_{\mathrm{G}}\right)$. Daraus resultieren die gewichteten Einwohneranteile $\left(\mathrm{e}_{\mathrm{L}} \mathrm{bzw} . \mathrm{e}_{\mathrm{G}}\right)$. Sie ergeben im Verhältnis zum tatsächlichen Einwohneranteil (e) die auf 1 normierten effektiven Einwohnergewichte $\left(\mathrm{v}_{\mathrm{L}}=\mathrm{e}_{\mathrm{L}} / \mathrm{e}\right.$ bzw. $\left.\mathrm{v}_{\mathrm{G}}=\mathrm{e}_{\mathrm{G}} / \mathrm{e}\right)$.

Zum Beispiel erhöht die Einwohnergewichtung der Stadtstaaten mit 135\% ihr effektives Einwohnergewicht bei den Ländersteuern 2020 auf ca. 131,6\% und reduziert das der Flächenländer auf ca. 97,5\%. Infolge der Einwohnergewichtungen der dünn besiedelten Flächenländer sinken die effektiven Einwohnergewichte der Stadtstaaten dort auf ca. 131,3\% und die der übrigen Flächenländer auf ca. 97,2\%.

Unter Berücksichtigung der effektiven Einwohnergewichte bei den Ländersteuern und den Gemeindesteuern gilt für die Ausgleichsmesszahl eines Landes:

$$
A M Z_{L}=v_{L} S M Z_{\varnothing L}+v_{G} g S M Z_{\varnothing G}
$$

Die Ausgleichsmesszahl entspricht prinzipiell der Summe der durchschnittlichen Steuerkraftmesszahlen der Ländersteuern (SMZ $\left.Z_{\varnothing \mathrm{L}}\right)$ und der mit dem Anteil (g) veranschlagten Gemeindesteuern (SMZ $\emptyset_{\mathrm{G}}$ ), allerdings modifiziert durch die normierten Einwohnergewichte der einzelnen Länder $\left(\mathrm{v}_{\mathrm{L}}\right.$ bzw. $\left.\mathrm{v}_{\mathrm{G}}\right)$.

Im Falle einer isolierten Variation der Steuerkraft eines Landes oder seiner Gemeinden verändert sich nach Gl. 1 die Finanzkraftmesszahl des Landes:

$$
\Delta \mathrm{FMZ}_{\mathrm{L}}=\Delta \mathrm{SMZ}_{\mathrm{L}}+\mathrm{g} \Delta \mathrm{SMZ}_{\mathrm{G}}
$$

Darüber hinaus verändert sich die Ausgleichsmesszahl des Landes, denn ein Anstieg der eigenen Steuerkraftmesszahlen verursacht nach Gl. 2 auch eine Erhöhung der durchschnittlichen Steuerkraftmesszahlen:

$$
\Delta \mathrm{AMZ}_{\mathrm{L}}=\mathrm{v}_{\mathrm{L}} \Delta \mathrm{SMZ} \mathrm{Z}_{\varnothing \mathrm{L}}+\mathrm{v}_{\mathrm{G}} \mathrm{g} \Delta \mathrm{SMZ} \mathrm{Z}_{\varnothing \mathrm{G}}
$$

Dabei hängt der Anstieg der durchschnittlichen Steuerkraftmesszahlen vom Anteil der ungewichteten Einwohner des Landes (e) an der Einwohnerzahl aller Länder ab. Wenn die Pro-Kopf-Steuerkraft eines großen Landes zunimmt, dann wächst die durchschnittliche Pro-Kopf-Steuerkraft aller Länder stärker als bei einem entsprechenden Anstieg in einem kleinen Land.

Für die Veränderung der durchschnittlichen Steuerkraftmesszahlen gilt daher:

$$
\begin{aligned}
\Delta \mathrm{SMZ}_{\varnothing \mathrm{L}} & =\mathrm{e} \Delta \mathrm{SMZ}_{\mathrm{L}}=\left(\mathrm{e}_{\mathrm{L}} / \mathrm{v}_{\mathrm{L}}\right) \Delta \mathrm{SMZ}_{\mathrm{L}} \\
\Delta \mathrm{SMZ}_{\varnothing \mathrm{G}} & =\mathrm{e} \Delta \mathrm{SMZ}_{\mathrm{G}}=\left(\mathrm{e}_{\mathrm{G}} / \mathrm{v}_{\mathrm{G}}\right) \Delta \mathrm{SMZ}_{\mathrm{G}}
\end{aligned}
$$

Einsetzen in Gl. 4 für die Veränderung der Ausgleichsmesszahl ergibt:

$$
\Delta \mathrm{AMZ}_{\mathrm{L}}=\mathrm{e}_{\mathrm{L}} \Delta \mathrm{SMZ}_{\mathrm{L}}+\mathrm{e}_{\mathrm{G}} \mathrm{g} \Delta \mathrm{SMZ}_{\mathrm{G}}
$$

Auf der ersten Stufe des Länderfinanzausgleichs wird die Differenz zwischen der Ausgleichs- und der Finanzkraftmesszahl nach Maßgabe des Ausgleichsatzes $(a=63 \%)$ teilweise kompensiert. Nach der Reform 2020 erfolgt der Finanzkraftaus- 
gleich nicht mehr durch horizontale Ausgleichszuweisungen und -beiträge, sondern durch $\mathrm{Zu}$ - und Abschläge $\left(\mathrm{Z}_{\mathrm{L}}\right)$ im Rahmen der Umsatzsteuerverteilung:

$$
\mathrm{Z}_{\mathrm{L}}=\mathrm{a}\left(\mathrm{AMZ} \mathrm{L}_{\mathrm{L}}-\mathrm{FMZ} \mathrm{L}_{\mathrm{L}}\right)
$$

Die Zu- und Abschläge eines Landes bei der Umsatzsteuerverteilung reagieren gemäß Gl. 7 auf die Veränderungen der Finanzkraftmesszahl und der Ausgleichsmesszahl. Eine Erhöhung der eigenen Steuerkraft senkt die Zuschläge bzw. erhöht die Abschläge bei der Umsatzsteuer. Daher bietet es sich an, diesen Effekt auf die negative Differenz von FMZ und AMZ zu beziehen:

$$
\Delta \mathrm{Z}_{\mathrm{L}}=-\mathrm{a}\left(\Delta \mathrm{FMZ}_{\mathrm{L}}-\Delta \mathrm{AMZ}_{\mathrm{L}}\right)=-\mathrm{a}\left[\left(1-\mathrm{e}_{\mathrm{L}}\right) \Delta \mathrm{SMZ}_{\mathrm{L}}+\left(1-\mathrm{e}_{\mathrm{G}}\right) \mathrm{g} \Delta \mathrm{SMZ}_{\mathrm{G}}\right]
$$

Nach Gl. 8 schlagen sich eigene Mehreinnahmen nicht im Umfang der erhöhten Finanzkraftmesszahl, sondern nur gebremst durch den entgegengerichteten Effekt der vergrößerten Ausgleichsmesszahl im Finanzkraftausgleich nieder. Dadurch werden die anderen Länder an der gewachsenen Steuerkraft eines Landes beteiligt. Auf den weiteren Stufen kann auch der Bund von der Erhöhung der Steuerkraft eines finanzschwachen Landes durch geringere Bundesergänzungszuweisungen profitieren.

Bei der Ermittlung der Grenzbelastungen geht es um die Auswirkungen einer isolierten Erhöhung der Steuerkraft eines Landes oder seiner Gemeinden auf die Zahlungen im Finanzausgleich ${ }^{4}$. Dabei bestimmen die direkten Finanzkrafteffekte die theoretischen bzw. unbereinigten Grenzbelastungen, die sich unter Vernachlässigung der Rückwirkungen auf die Ausgleichsmesszahlen ergeben. Die effektiven Grenzbelastungen liegen stets darunter, weil ein Anstieg der Steuerkraft eines Landes die durchschnittliche Steuerkraft aller Länder und damit auch die Ausgleichsmesszahl des Landes selbst erhöht.

Aus Gl. 8 lassen sich die Grenzbelastungen des Landeshaushalts $\left(\mathrm{GB}_{\mathrm{L}}\right)$ und des Gesamthaushalts $\left(\mathrm{GB}_{\mathrm{LG}}\right)$ bestimmen. Die Grenzbelastungen sind allgemein definiert als Relation zwischen der mit negativem Vorzeichen versehenen Veränderung der $\mathrm{Zu}$ - und Abschläge bei der Umsatzsteuer $\left(-\Delta \mathrm{Z}_{\mathrm{L}}\right)$ und der Veränderung der Landeseinnahmen $\left(\Delta \mathrm{SMZ}_{\mathrm{L}}\right)$ bzw. der Gesamteinnahmen $\left(\Delta \mathrm{SMZ}_{\mathrm{L}}+\Delta \mathrm{SMZ}_{\mathrm{G}}\right)$.

Nur das Aufkommen der Ländersteuern betrifft den Landeshaushalt direkt:

$$
\mathrm{GB}_{\mathrm{L}}=\mathrm{a} \frac{\left(1-\mathrm{e}_{\mathrm{L}}\right) \Delta \mathrm{SMZ}_{\mathrm{L}}+\left(1-\mathrm{e}_{\mathrm{G}}\right) \mathrm{g} \Delta \mathrm{SMZ}_{\mathrm{G}}}{\Delta \mathrm{SMZ} \mathrm{Z}_{\mathrm{L}}}
$$

Im Gesamthaushalt ist auch das Aufkommen der Gemeindesteuern relevant:

$$
\mathrm{GB}_{\mathrm{LG}}=\mathrm{a} \frac{\left(1-\mathrm{e}_{\mathrm{L}}\right) \Delta \mathrm{SMZ}_{\mathrm{L}}+\left(1-\mathrm{e}_{\mathrm{G}}\right) \mathrm{g} \Delta \mathrm{SMZ}_{\mathrm{G}}}{\Delta \mathrm{SMZ}_{\mathrm{L}}+\Delta \mathrm{SMZ}_{\mathrm{G}}}
$$

Auf der Basis der Gln. 9 und 10 lassen sich die Grenzbelastungen konkreter Steuern im Finanzkraftausgleich genauer bestimmen (vgl. Kap. 4). Zuvor werden aber

\footnotetext{
${ }^{4}$ Die in der Literatur oft verwendeten absoluten Verbleibsbeträge oder relativen Verbleibsquoten (VQ) sind die Kehrseite der Grenzbelastungen (GB). Sie geben an, welcher Teil der Steuermehreinnahmen im Landes- oder Gesamthaushalt verbleibt: $\mathrm{VQ}=1-\mathrm{GB}$.
} 
die Bundesergänzungszuweisungen analysiert, die zusätzliche Grenzbelastungen bei den Empfängern erzeugen.

\section{Bundesergänzungszuweisungen}

Der Bund erhöht die Finanzkraft der Länder durch allgemeine Bundesergänzungszuweisungen sowie Bundesergänzungszuweisungen für Sonderbedarfe. Letztere sind im vorliegenden Kontext nur relevant, soweit sie von der Steuerkraft abhängen und damit Grenzbelastungen erzeugen. Das betrifft allein die neuen Bundesergänzungszuweisungen für unterproportionale Gemeindesteuerkraft.

Die allgemeinen Bundesergänzungszuweisungen $\left(\mathrm{BEZ}_{\mathrm{A}}\right)$ setzen den horizontalen Finanzkraftausgleich unter den Ländern mit einem vertikalen Instrument fort. Relativ finanzschwache Länder erhalten zusätzliche Bundesmittel zur Deckung ihres Finanzbedarfs. Damit wird die Lücke bis zu 99,75\% des Länderdurchschnitts, die nach Finanzkraftausgleich noch verbleibt, zu $80 \%$ geschlossen. Maßgeblich für die allgemeinen Bundesergänzungszuweisungen ist einerseits der Ausgleichsatz $(b=80 \%)$ und andererseits die Differenz zwischen einem bestimmten Prozentsatz $\left(b^{\circ}=99,75 \%\right)$ der Ausgleichsmesszahl und der Finanzkraftmesszahl nach Finanzkraftausgleich $\left(\mathrm{FMZ}_{\mathrm{L} 2}\right)$ :

$$
\mathrm{BEZ}_{\mathrm{A}}=\mathrm{b}\left(\mathrm{b}^{\mathrm{o}} \mathrm{AMZ} \mathrm{L}_{\mathrm{L}}-\mathrm{FMZ}_{\mathrm{L} 2}\right) \quad \text { wenn } \quad \mathrm{FMZ}_{\mathrm{L} 2}<\mathrm{b}^{\mathrm{o}} \mathrm{AMZ} \mathrm{L}_{\mathrm{L}}
$$

Die Finanzkraftmesszahl nach Finanzkraftausgleich entspricht der Finanzkraftmesszahl vor Finanzkraftausgleich zuzüglich der bereits erhaltenen Umsatzsteuerzuschläge:

$$
\mathrm{FMZ}_{\mathrm{L} 2}=\mathrm{FMZ}_{\mathrm{L}}+\mathrm{a}\left(\mathrm{AMZ}_{\mathrm{L}}-\mathrm{FMZ}_{\mathrm{L}}\right)
$$

Somit gilt für die allgemeinen Bundesergänzungszuweisungen:

$$
\begin{aligned}
\mathrm{BEZ}_{\mathrm{A}} & =\mathrm{b}\left[\left(\mathrm{b}^{\mathrm{o}}-\mathrm{a}\right) \mathrm{AMZ}_{\mathrm{L}}-(1-\mathrm{a}) \mathrm{FMZ}_{\mathrm{L}}\right] \text { wenn } \mathrm{FMZ}_{\mathrm{L}}<\mathrm{r}^{\mathrm{o}} \mathrm{AMZ} \mathrm{L}_{\mathrm{L}} \text { mit } \mathrm{r}^{\mathrm{o}} \\
& =\left(\mathrm{b}^{\mathrm{o}}-\mathrm{a}\right) /(1-\mathrm{a})
\end{aligned}
$$

Die Begrenzung auf einen Prozentsatz $b^{\circ}$ der Ausgleichsmesszahl, der kleiner als $100 \%$ ist, hat zur Folge, dass allgemeine Bundesergänzungszuweisungen nur an Länder gezahlt werden, deren Finanzkraftmesszahl vor Finanzkraftausgleich einen bestimmten Prozentsatz $r^{o}$ der Ausgleichsmesszahl unterschreitet. Dabei gilt $r^{\circ}<b^{o}$ für $\mathrm{a}>0$.

Unter Berücksichtigung der Gl. 3 für die Veränderung der Finanzkraftmesszahl und (6) für die Veränderung der Ausgleichsmesszahl lässt sich aus Gl. 13 die Veränderung der Bundesergänzungszuweisungen infolge einer Variation der Länder- oder Gemeindesteuerkraft ermitteln: 


$$
\Delta B E Z_{\mathrm{A}}=-\mathrm{b}(1-\mathrm{a})\left[\left(1-\mathrm{r}^{\mathrm{o}} \mathrm{e}_{\mathrm{L}}\right) \Delta \mathrm{SMZ}_{\mathrm{L}}+\left(1-\mathrm{r}^{\mathrm{o}} \mathrm{e}_{\mathrm{G}}\right) \mathrm{g} \Delta \mathrm{SMZ}_{\mathrm{G}}\right]
$$

Daraus resultieren die Grenzbelastungen bei den allgemeinen Bundesergänzungszuweisungen für den Landeshaushalt und den Gesamthaushalt, die zusätzlich zu den Grenzbelastungen im Finanzkraftausgleich eintreten:

$$
\begin{aligned}
& \mathrm{GB}_{\mathrm{L}}=\mathrm{b}(1-\mathrm{a}) \frac{\left(1-\mathrm{r}^{\mathrm{o}} \mathrm{e}_{\mathrm{L}}\right) \Delta \mathrm{SMZ}_{\mathrm{L}}+\left(1-\mathrm{r}^{\mathrm{o}} \mathrm{e}_{\mathrm{G}}\right) \mathrm{g} \Delta \mathrm{SMZ}_{\mathrm{G}}}{\Delta \mathrm{SMZ}} \\
& \mathrm{GB}_{\mathrm{LG}}=\mathrm{b}(1-\mathrm{a}) \frac{\left(1-\mathrm{r}^{\mathrm{o}} \mathrm{e}_{\mathrm{L}}\right) \Delta \mathrm{SMZ}_{\mathrm{L}}+\left(1-\mathrm{r}^{\mathrm{o}} \mathrm{e}_{\mathrm{G}}\right) \mathrm{g} \Delta \mathrm{SMZ}_{\mathrm{G}}}{\Delta \mathrm{SMZ}_{\mathrm{L}}+\Delta \mathrm{SMZ}_{\mathrm{G}}}
\end{aligned}
$$

Eine formale Aggregation der Grenzbelastungen im Finanzkraftausgleich und bei den Bundesergänzungszuweisungen ist nicht erforderlich, da sich die gesamten Grenzbelastungen durch einfache Addition der Teileffekte ergeben. Der gesonderte Ausweis der Grenzbelastungen bei den Bundesergänzungszuweisungen erklärt zudem transparent die Differenzen zwischen den gesamten Grenzbelastungen der einzelnen Länder.

Infolge der neuen Bundesergänzungszuweisungen für unterproportionale Gemeindesteuerkraft $\left(\mathrm{BEZ}_{\mathrm{G}}\right)$ treten für die Empfängerländer weitere Grenzbelastungen ein, weil auch diese Zuweisungen auf eine Veränderung der eigenen Steuerkraft reagieren. Die Bezugsgrößen weichen jedoch von denen der übrigen Ausgleichsinstrumente ab, was zu unsystematischen Ausgleichseffekten führt (Scherf 2020, S. 10-12). Maßgeblich sind hier die Differenzen zwischen der kommunalen Steuerkraft pro Einwohner in einem Land $\left(\mathrm{SMZ}_{\mathrm{G}}\right)$ und der mittleren Gemeindesteuerkraft im Bundesgebiet $\left(\mathrm{SMZ}_{\emptyset \mathrm{G}}\right)$. Letztere dient im vorliegenden Fall als Ausgleichsmesszahl. Wenn die Steuerkraftmesszahl unter einem bestimmten Prozentsatz $\left(c^{\circ}=80 \%\right)$ der Ausgleichsmesszahl liegt, werden die Fehlbeträge nach Maßgabe des Ausgleichsatzes $(c=53,5 \%)$ kompensiert:

$$
\mathrm{BEZ}_{\mathrm{G}}=\mathrm{c}\left(\mathrm{c}^{\mathrm{o}} \mathrm{SMZ} \mathrm{CG}_{\mathrm{G}}-\mathrm{SMZ}_{\mathrm{G}}\right) \text { wenn } \mathrm{SMZ}_{\mathrm{G}}<\mathrm{c}^{\mathrm{o}} \mathrm{AMZ} \mathrm{Z}_{\mathrm{G}}
$$

Nach Gl. 6 verändert sich die durchschnittliche Steuerkraft der Gemeinden mit der Gemeindesteuerkraft eines Landes $\left(\Delta S M Z_{\emptyset \mathrm{G}}=\mathrm{e} \Delta \mathrm{SMZ}_{\mathrm{G}}\right)$. Daher gilt für die Veränderungen der Bundesergänzungszuweisungen nach der Gemeindefinanzkraft:

$$
\Delta \mathrm{BEZ}_{\mathrm{G}}=\mathrm{c}\left(\mathrm{c}^{\mathrm{o}} \Delta \mathrm{SMZ} \mathrm{Z}_{\varnothing \mathrm{G}}-\Delta \mathrm{SMZ} \mathrm{Z}_{\mathrm{G}}\right)=-\mathrm{c}\left(1-\mathrm{c}^{\mathrm{o}} \mathrm{e}\right) \Delta \mathrm{SMZ} \mathrm{Z}_{\mathrm{G}}
$$

Daraus lassen sich die zusätzlichen Grenzbelastungen der Empfängerländer ableiten:

$$
\begin{aligned}
& \mathrm{GB}_{\mathrm{L}}=\mathrm{c}\left(1-\mathrm{c}^{\mathrm{o}} \mathrm{e}\right) \Delta \mathrm{SMZ}_{\mathrm{G}} / \Delta \mathrm{SMZ}_{\mathrm{L}} \\
& \mathrm{GB}_{\mathrm{LG}}=\mathrm{c}\left(1-\mathrm{c}^{\mathrm{e}}\right) \Delta \mathrm{SMZ}_{\mathrm{G}} /\left(\Delta \mathrm{SMZ}_{\mathrm{L}}+\Delta \mathrm{SMZ}_{\mathrm{G}}\right)
\end{aligned}
$$

Damit stehen alle Komponenten für die Ermittlung der aggregierten Grenzbelastungen der Länder im Länderfinanzausgleich einschließlich Bundesergänzungszuweisungen zur Verfügung. Die aggregierten Grenzbelastungen unterscheiden sich 
nach drei Ländergruppen: (1) Empfänger von Bundesergänzungszuweisungen für unterproportionale Gemeindesteuerkraft, (2) Empfänger von allgemeinen Bundesergänzungszuweisungen und (3) übrige Länder.

Alle Komponenten zeichnen sich dadurch aus, dass die theoretischen bzw. unbereinigten Grenzbelastungen stets über den Werten liegen, die sich unter Berücksichtigung der systemimmanenten Rückkoppelungseffekte tatsächlich einstellen. Das Ausmaß der Abweichungen variiert mit den Einwohneranteilen der einzelnen Länder, wobei für den Finanzkraftausgleich und die allgemeinen Bundesergänzungszuweisungen nach Ländersteuern und Gemeindesteuern differenzierte gewichtete Einwohneranteile herangezogen werden müssen.

\section{Grenzbelastungen einzelner Steuern}

Auf der Basis der abgeleiteten allgemeinen Zusammenhänge werden im Folgenden die Grenzbelastungen einzelner Steuern aufgezeigt. Darüber hinaus lässt sich das Instrumentarium auch zur Analyse verschiedener Reformoptionen verwenden.

\subsection{Körperschaftsteuer}

Die Körperschaftsteuer ist eine Gemeinschaftsteuer, an der neben dem Bund nur die Länder beteiligt sind. Der Anteil des Bundes spielt für die Grenzbelastungen aber keine Rolle, weil seine Einnahmen nicht zur Bezugsgröße rechnen. Insofern genügt hier - wie bei reinen Ländersteuern (z. B. Erbschaftsteuer) - die Bezugnahme auf den Landeshaushalt. Die Mehreinnahmen $\left(\Delta S M Z_{L}>0\right)$ entsprechen denen im Gesamthaushalt, weil die Gemeinden keine zusätzlichen Einnahmen erhalten $\left(\triangle \mathrm{SMZ}_{\mathrm{G}}=0\right)$. Daher bleiben auch die Bundesergänzungszuweisungen nach der Gemeindesteuerkraft unberührt.

Für Ländersteuern und die Körperschaftsteuer ergeben sich nach den Gln. 9 und 15 folgende Grenzbelastungen durch den Finanzkraftausgleich (FKA) bzw. die allgemeinen Bundesergänzungszuweisungen:

$$
\begin{aligned}
\mathrm{GB}_{\mathrm{L}} & =\mathrm{GB}_{\mathrm{LG}}=\mathrm{a}\left(1-\mathrm{e}_{\mathrm{L}}\right) \quad \text { durch FKA } \\
\mathrm{GB}_{\mathrm{L}} & =\mathrm{GB}_{\mathrm{LG}}=\mathrm{b}(1-\mathrm{a})\left(1-\mathrm{r}^{\mathrm{O}} \mathrm{e}_{\mathrm{L}}\right) \quad \text { durch } \mathrm{BEZ}_{\mathrm{A}}
\end{aligned}
$$

Abb. 1 zeigt die Grenzbelastungen der einzelnen Länder geordnet nach der aggregierten Grenzbelastung $\left(\sum \mathrm{GB}_{\mathrm{L}}\right)$. Für relativ kleine Bundesländer liegen die Werte nahe bei der unbereinigten Grenzbelastung $\left(\mathrm{GB}_{\mathrm{U}}\right)$. Stärkere Abweichungen finden sich dagegen bei den großen Bundesländern NW, BY und BW. Die Grenzbelastungen sind bei den relativ finanzstarken Ländern moderat, erreichen aber bei den relativ finanzschwachen Ländern schon Werte knapp unter bzw. über $90 \%$. Von einem Euro Mehraufkommen können diese Länder also netto nur rund 10 Cent in ihrem Haushalt verbuchen. 


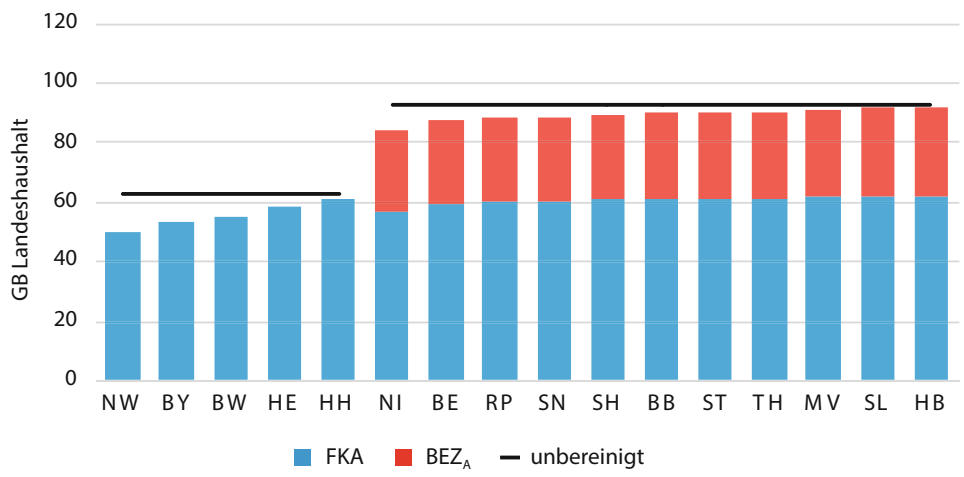

\begin{tabular}{|l|rrrrrrrrrrrrrrrr|r|}
\hline Land & NW & BY & BW & HE & HH & NI & BE & RP & SN & SH & BB & ST & TH & MV & SL & HB & GB \\
\hline FKA & 49,7 & 53,3 & 54,8 & 58,4 & 61,2 & 57,1 & 59,4 & 60,0 & 60,0 & 60,9 & 61,1 & 61,4 & 61,4 & 61,8 & 62,3 & 62,3 & 63,0 \\
BEZ $_{A}$ & & & & & & 26,8 & 27,9 & 28,2 & 28,2 & 28,6 & 28,7 & 28,8 & 28,9 & 29,0 & 29,3 & 29,3 & 29,6 \\
\hline$\sum \mathrm{GB}_{\mathrm{L}}$ & 49,7 & 53,3 & 54,8 & 58,4 & 61,2 & 83,9 & 87,3 & 88,2 & 88,2 & 89,5 & 89,9 & 90,2 & 90,3 & 90,9 & 91,5 & 91,6 & 92,6 \\
\hline
\end{tabular}

Abb. 1 Grenzbelastungen bei der Körperschaftsteuer

\subsection{Lohnsteuer}

Komplexer sind die Formeln für die Grenzbelastungen, wenn Länder und Gemeinden am Aufkommen einer Gemeinschaftsteuer beteiligt sind (Lohn- und Einkommensteuer, Abgeltungsteuer, Gewerbesteuer). Hier fließen Einnahmen an Land und Gemeinden, wobei die Gemeindeeinnahmen anteilig zur Finanzkraft des Landes rechnen. Da sich die Mehreinnahmen auf Land und Gemeinden verteilen, divergieren die Grenzbelastungen bezogen auf den Landeshaushalt und den Gesamthaushalt.

Bei solchen Steuern treten Veränderungen der Steuerkraft des Landes und seiner Gemeinden simultan ein. Dabei entspricht die Relation zwischen der Steuerkraftänderung der Gemeinden und der Steuerkraftänderung des Landes der Beteiligungsquote der Gemeinden $\left(b_{G}\right)$ relativ zur Beteiligungsquote des Landes $\left(b_{L}\right)$ an der Gemeinschaftsteuer: ${ }^{5}$

$$
\Delta \mathrm{SMZ}_{\mathrm{G}} / \Delta \mathrm{SMZ}_{\mathrm{L}}=\mathrm{b}_{\mathrm{G}} / \mathrm{b}_{\mathrm{L}}
$$

Nach Substitution von $\Delta \mathrm{SMZ}_{\mathrm{G}}$ durch $\left(\mathrm{b}_{\mathrm{G}} / \mathrm{b}_{\mathrm{L}}\right) \Delta \mathrm{SMZ} \mathrm{Z}_{\mathrm{L}}$ in den allgemeinen Gln. 9, 15 und 19 für die Grenzbelastungen des Landeshaushalts ergeben sich folgende Grenzbelastungen auf den einzelnen Stufen des Länderfinanzausgleichs:

$$
\begin{aligned}
& \mathrm{GB}_{\mathrm{L}}=\mathrm{a}\left[\left(1-\mathrm{e}_{\mathrm{L}}\right)+\left(1-\mathrm{e}_{\mathrm{G}}\right) \mathrm{g}\left(\mathrm{b}_{\mathrm{G}} / \mathrm{b}_{\mathrm{L}}\right)\right] \text { durch FKA } \\
& \mathrm{GB}_{\mathrm{L}}=\mathrm{b}(1-\mathrm{a})\left[\left(1-\mathrm{r}^{\mathrm{o}} \mathrm{e}_{\mathrm{L}}\right)+\left(1-\mathrm{r}^{\mathrm{o}} \mathrm{e}_{\mathrm{G}}\right) \mathrm{g}\left(\mathrm{b}_{\mathrm{G}} / \mathrm{b}_{\mathrm{L}}\right)\right] \text { durch BEZ }
\end{aligned}
$$

\footnotetext{
5 Diese Relation beträgt bei der Lohnsteuer 15/42,5 und bei der Abgeltungsteuer 12/44. Bei der Gewerbesteuer liegt sie mit 4,375/91,25 weit darunter. Wie noch gezeigt wird, unterscheidet sich die Gewerbesteuer grundlegend von den beiden anderen, weil sie in erster Linie eine Gemeindesteuer darstellt.
} 


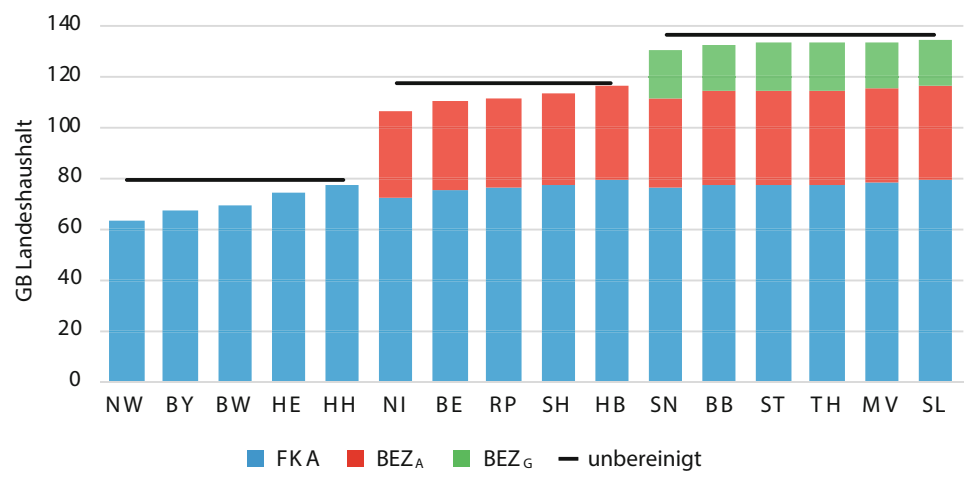

\begin{tabular}{|c|c|c|c|c|c|c|c|c|c|c|c|c|c|c|c|c|c|}
\hline Land & JW & BY & BW & $\mathrm{HE}$ & $\mathrm{HH}$ & $\mathrm{NI}$ & $B E$ & RP & SH & $\mathrm{HB}$ & SN & BB & ST & $\mathrm{TH}$ & MV & $S L$ & $\mathrm{~GB}_{\mathrm{U}}$ \\
\hline FKA & 62,9 & 67,4 & 69,3 & 73,8 & 77,4 & 72,2 & 75,1 & 75,9 & 77,0 & 78,8 & 75,9 & 77,3 & 77,6 & 77,7 & 78,2 & 78,8 & 79,7 \\
\hline$B E Z_{A}$ & & & & & & 34,0 & 35,3 & 35,7 & 36,2 & 37,0 & 35,7 & 36,3 & 36,5 & 36,5 & 36,7 & 37,0 & 37,4 \\
\hline $\mathrm{BEZ}_{\mathrm{G}}$ & & & & & & & & & & & 18,1 & 18,4 & 18,5 & 18,5 & 18,6 & 18,7 & 18,9 \\
\hline$\sum \mathrm{GB}_{\mathrm{L}}$ & 62,9 & 67,4 & 69,3 & 73,8 & 77,4 & 106,2 & 110,4 & 111,5 & 113,1 & 115,8 & 129,7 & 132,1 & 132,6 & 132,7 & 133,5 & 134,5 & 136,0 \\
\hline FKA & 46,5 & 49,8 & 51,2 & 54,6 & 57,2 & 53,4 & 55,5 & 56,1 & 56,9 & 58,3 & 56,1 & 57,1 & 57,4 & 57,4 & 57,8 & 58,2 & 58,9 \\
\hline$B E Z_{A}$ & & & & & & 25,1 & 26,1 & 26,4 & 26,7 & 27,4 & 26,4 & 26,9 & 27,0 & 27,0 & 27,1 & 27,4 & 27,7 \\
\hline$B E Z_{G}$ & & & & & & & & & & & 13,4 & 13,6 & 13,7 & 13,7 & 13,7 & 13,8 & 14,0 \\
\hline$\sum \mathrm{GB}_{\mathrm{LG}}$ & 46,5 & 49,8 & 51,2 & 54,6 & 57,2 & 78,5 & 81,6 & 82,4 & 83,6 & 85,6 & 95,8 & 97,6 & 98,0 & 98,1 & 98,7 & 99,4 & 100,5 \\
\hline
\end{tabular}

Abb. 2 Grenzbelastungen bei der Lohnsteuer

$$
\mathrm{GB}_{\mathrm{L}}=\mathrm{c}\left(1-\mathrm{c}^{\mathrm{o}} \mathrm{q}\right)\left(\mathrm{b}_{\mathrm{G}} / \mathrm{b}_{\mathrm{L}}\right) \quad \text { durch BEZ } \mathrm{G}_{\mathrm{G}}
$$

Die Grenzbelastungen des Landeshaushalts haben jeweils die Form $\mathrm{GB}_{\mathrm{L}}=-\Delta \mathrm{X} /$ $\Delta \mathrm{SMZ}_{\mathrm{L}}$ mit $\Delta \mathrm{X} \in\left(\Delta \mathrm{Z}_{\mathrm{L}}, \Delta \mathrm{BEZ}_{\mathrm{A}}, \Delta \mathrm{BEZ}_{\mathrm{G}}\right)$. Unter Verwendung der Beteiligungsquoten der Gemeinden und des Landes lassen sich die Grenzbelastungen des Gesamthaushalts auf allen Stufen daher einfach aus den Grenzbelastungen des Landeshaushalts ableiten:

$$
\mathrm{GB}_{\mathrm{LG}}=\mathrm{GB}_{\mathrm{L}}\left[\Delta \mathrm{SMZ}_{\mathrm{L}} /\left(\Delta \mathrm{SMZ}_{\mathrm{L}}+\Delta \mathrm{SMZ}_{\mathrm{G}}\right)\right]=\mathrm{GB}_{\mathrm{L}} /\left(1+\mathrm{b}_{\mathrm{G}} / \mathrm{b}_{\mathrm{L}}\right)
$$

Abb. 2 dokumentiert das hohe Niveau der Grenzbelastungen bei der quantitativ gewichtigen Lohnsteuer. In den Landeshaushalten der Länder, die Bundesergänzungszuweisungen erhalten, führen zusätzliche Lohnsteuereinnahmen zu einer Nettobelastung. Besonders betroffen sind die Empfänger von Bundesergänzungszuweisungen für unterproportionale Gemeindesteuerkraft. Auch die Grenzbelastungen des Gesamthaushalts erreichen hohe Werte, die in der Spitze eine Vollabschöpfung bedeuten.

\subsection{Abgeltungsteuer}

Auf einem etwas tieferen Niveau liegen die Grenzbelastungen des Landeshaushalts bei der Abgeltungsteuer (Abb. 3). Die Differenz beruht auf der kleineren Relation $b_{G} / b_{L}$. Der Anteil der Mehreinnahmen, der in den Landeshaushalt fließt, ist bei der 


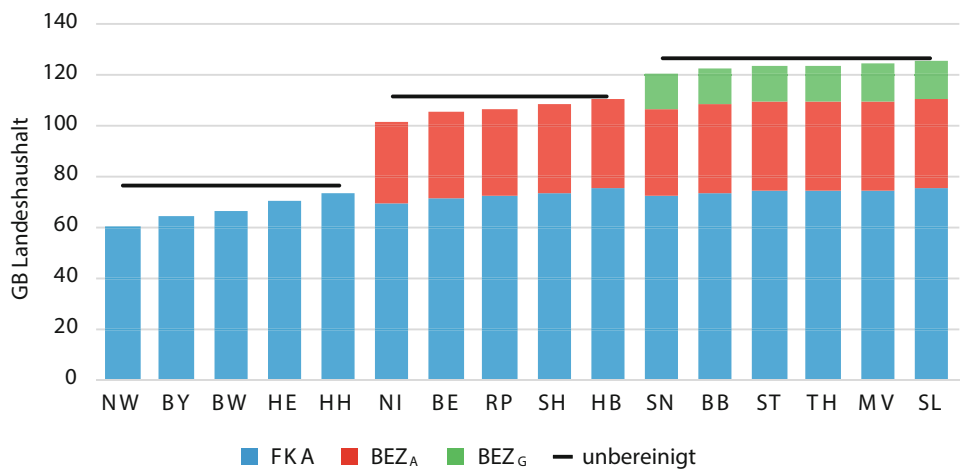

\begin{tabular}{|l|rrrrrrrrrrrrrrrr|r|}
\hline Land & NW & BY & BW & HE & HH & NI & BE & RP & SH & HB & SN & BB & ST & TH & MV & SL & G $B_{U}$ \\
\hline FKA & 59,9 & 64,2 & 66,0 & 70,3 & 73,7 & 68,8 & 71,5 & 72,2 & 73,3 & 75,1 & 72,3 & 73,6 & 73,9 & 74,0 & 74,4 & 75,0 & 75,9 \\
BE $Z_{A}$ & & & & & & 32,3 & 33,6 & 34,0 & 34,5 & 35,3 & 34,0 & 34,6 & 34,7 & 34,8 & 35,0 & 35,2 & 35,7 \\
BE $Z_{G}$ & & & & & & & & & & & 14,0 & 14,2 & 14,3 & 14,3 & 14,4 & 14,5 & 14,6 \\
\hline$\sum \mathrm{GB}_{\mathrm{L}}$ & 59,9 & 64,2 & 66,0 & 70,3 & 73,7 & 101,1 & 105,1 & 106,2 & 107,8 & 110,3 & 120,2 & 122,5 & 122,9 & 123,0 & 123,8 & 124,7 & 126,1 \\
\hline FKA & 47,1 & 50,5 & 51,9 & 55,2 & 57,9 & 54,0 & 56,2 & 56,8 & 57,6 & 59,0 & 56,8 & 57,9 & 58,1 & 58,1 & 58,5 & 58,9 & 59,6 \\
BE $Z_{A}$ & & & & & & 25,4 & 26,4 & 26,7 & 27,1 & 27,7 & 26,7 & 27,2 & 27,3 & 27,3 & 27,5 & 27,7 & 28,0 \\
BE $Z_{G}$ & & & & & & & & & & & 11,0 & 11,2 & 11,2 & 11,2 & 11,3 & 11,4 & 11,5 \\
\hline$\sum \mathrm{SB}_{\mathrm{LG}}$ & 47,1 & 50,5 & 51,9 & 55,2 & 57,9 & 79,4 & 82,6 & 83,4 & 84,7 & 86,7 & 94,5 & 96,2 & 96,6 & 96,7 & 97,3 & 98,0 & 99,1 \\
\hline
\end{tabular}

Abb. 3 Grenzbelastungen bei der Abgeltungsteuer

Abgeltungsteuer größer, der Anteil, der in den Gemeindehaushalt fließt und dem Land zugerechnet wird, ist dagegen kleiner als bei der Lohnsteuer.

Da zugleich der Faktor $\left(b_{G} / b_{L}\right) /\left(1+b_{G} / b_{L}\right)$ steigt, fallen die Grenzbelastungen des Gesamthaushalts für alle Länder mit Ausnahme der Bezieher von Bundesergänzungszuweisungen für unterproportionale Gemeindesteuerkraft etwas größer aus als bei der Lohnsteuer. Letztere profitieren von dem geringeren Gemeindeanteil an den Mehreinnahmen. Er sorgt dafür, dass die Grenzbelastungen auf der letzten Stufe der Gemeindesteuerkraftzuweisungen deutlich geringer ausfallen als bei der Lohnsteuer, was den Anstieg auf den beiden vorgelagerten Stufen überkompensiert.

\subsection{Grundsteuer}

Die Grundsteuer ist eine reine Gemeindesteuer. Das Land erhält keine zusätzlichen Einnahmen $\left(\triangle S M Z_{L}=0\right)$, wird aber durch die anteilige Anrechnung der Gemeindesteuern im Finanzausgleich finanziell belastet. Deshalb kann die Grenzbelastung sinnvoll nur auf den Gesamthaushalt von Land und Gemeinden bezogen werden, in dem sich die Mehreinnahmen der Gemeinden $\left(\Delta \mathrm{SMZ}_{\mathrm{G}}>0\right)$ niederschlagen. Auch die Bundesergänzungszuweisungen für unterproportionale Gemeindesteuerkraft sind hier relevant.

Unter Berücksichtigung der Gln. 10, 16 und 20 gilt für Gemeindesteuern:

$$
\mathrm{GB}_{\mathrm{LG}}=\mathrm{a}\left(1-\mathrm{e}_{\mathrm{G}}\right) \mathrm{g} \text { durch FKA }
$$




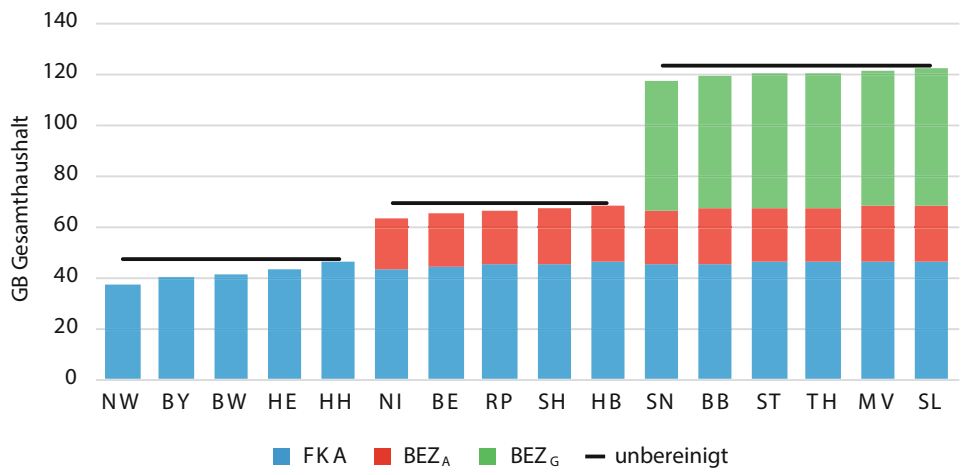

\begin{tabular}{|l|rrrrrrrrrrrrrrrr|r|}
\hline Land & NW & BY & BW & HE & HH & NI & BE & RP & SH & HB & SN & BB & ST & TH & MV & SL & G $B_{U}$ \\
\hline FKA & 37,3 & 40,0 & 41,1 & 43,8 & 45,9 & 42,8 & 44,5 & 45,0 & 45,6 & 46,7 & 45,0 & 45,8 & 46,0 & 46,1 & 46,3 & 46,7 & 47,3 \\
BE $_{A}$ & & & & & & 20,1 & 20,9 & 21,1 & 21,5 & 22,0 & 21,1 & 21,5 & 21,6 & 21,6 & 21,8 & 21,9 & 22,2 \\
BE $_{G}$ & & & & & & & & & & & 51,4 & 52,2 & 52,4 & 52,4 & 52,7 & 53,0 & 53,5 \\
\hline$\sum \mathrm{GB}_{\mathrm{LG}}$ & 37,3 & 40,0 & 41,1 & 43,8 & 45,9 & 63,0 & 65,5 & 66,1 & 67,1 & 68,7 & 117,5 & 119,6 & 120,0 & 120,1 & 120,8 & 121,6 & 123,0 \\
\hline$\sum \mathrm{GB}_{\mathrm{H}}$ & 30,9 & 47,9 & 48,9 & 43,7 & 40,1 & 69,0 & 38,2 & 77,7 & 80,7 & 74,0 & 111,6 & 138,3 & 135,2 & 130,3 & 133,7 & 131,0 & 123,0 \\
$\Delta \mathrm{GB}$ & $-6,4$ & 7,9 & 7,7 & $-0,1$ & $-5,7$ & 6,0 & $-27,3$ & 11,6 & 13,6 & 5,3 & $-6,0$ & 18,7 & 15,2 & 10,2 & 13,0 & 9,4 & 0,0 \\
$\mathrm{H} / \mathrm{H}_{\varnothing}$ & 120,8 & 83,5 & 84,1 & 100,2 & 114,3 & 91,3 & 171,5 & 85,1 & 83,1 & 145,5 & 105,4 & 86,5 & 88,8 & 92,2 & 90,3 & 92,8 & 100,0 \\
\hline
\end{tabular}

Abb. 4 Grenzbelastungen bei der Grundsteuer

$$
\begin{aligned}
\mathrm{GB}_{\mathrm{LG}} & =\mathrm{b}(1-\mathrm{a})\left(1-\mathrm{r}^{\mathrm{o}} \mathrm{e}_{\mathrm{G}}\right) \mathrm{g} \quad \text { durch } \mathrm{BEZ}_{\mathrm{A}} \\
\mathrm{GB}_{\mathrm{LG}} & =\mathrm{c}\left(1-\mathrm{c}^{\mathrm{o}} \mathrm{q}\right) \quad \text { durch } \mathrm{BEZ}_{\mathrm{G}}
\end{aligned}
$$

Abb. 4 illustriert den enormen Einfluss der Bundesergänzungszuweisungen für unterproportionale Gemeindesteuerkraft auf die Grenzbelastungen. Die Empfängerländer verzeichnen einen Anstieg der Grenzbelastungen um mehr als 50 Prozentpunkte und verlieren im Gesamthaushalt $17,5 \%$ bis $21,6 \%$ ihrer Mehreinnahmen. Sie wären also finanziell besser gestellt, wenn ihre Steuerkraft nicht wachsen würde. Anreizfeindlicher kann man ein Finanzausgleichssystem nicht gestalten.

Bei der Grundsteuer basieren die Grenzbelastungen auf der Annahme, dass jedes Land den bundesdurchschnittlichen Hebesatz verwendet. Dann entspricht die Verteilung der tatsächlichen Einnahmen auf die Länder der Verteilung der Grundbeträge, die im Finanzausgleich als Maßstab der Realsteuerkraft dienen. Vom Bundesdurchschnitt abweichende landesdurchschnittliche Hebesätze sorgen aber dafür, dass die tatsächlichen Einnahmen bei einem Anstieg der Steuerkraft (gemessen an den Grundbeträgen) nicht überall gleich stark zunehmen. In Ländern mit unterdurchschnittlichen Hebesätzen fallen die Grenzbelastungen bezogen auf ihre tatsächlichen Mehreinnahmen noch höher aus, während sie in Ländern mit überdurchschnittlichen Hebesätzen zurückgehen.

Abb. 4 zeigt im unteren Teil das beachtliche Ausmaß der Belastungsdifferenzen unter Verwendung der landesdurchschnittlichen Hebesätze des Jahres 2018 für die Grundsteuer B (Statistisches Bundesamt 2019). Die aggregierten Grenzbelastungen berechnet zu tatsächlichen Hebesätzen $\left(\sum \mathrm{GB}_{\mathrm{H}}\right)$ liegen um bis zu 18,7 Prozentpunkte (BB) über bzw. bis zu 27,3 Prozentpunkte (BE) unter den Grenzbelastungen 
zum normierten Hebesatz. ${ }^{6}$ Das spricht aber nicht gegen die Verwendung des bundesdurchschnittlichen Hebesatzes zur Bestimmung der Realsteuerkraft. Diese Vorgehensweise sorgt dafür, dass hebesatzbedingte Mehr- oder Mindereinnahmen, die in der Finanzautonomie der Länder liegen, keinen Einfluss auf ihre Zuweisungen bzw. Abschöpfungen haben.

\subsection{Gewerbesteuer}

Die Gewerbesteuer ist zwar faktisch eine Gemeinschaftsteuer, deren Aufkommen über die Gewerbesteuerumlage teils dem Bund und den Ländern zufließt. Bezüglich der hier relevanten Landes- und Gemeindeanteile erfolgt durch die Umlage aber nur eine Umverteilung der im Land anfallenden Mittel. Sie beeinflusst dennoch den Finanzausgleich, vor allem, weil die Einnahmen des Landes vollständig, die Einnahmen der Gemeinden aber nur anteilig angerechnet werden. Weniger bedeutsam sind demgegenüber die unterschiedlichen Einwohnergewichte auf der Landes- und Gemeindeebene.

Die Grenzbelastungen errechnen sich nach den Gln. 24 bis 27 für Gemeinschaftsteuern. Allerdings können die Grenzbelastungen bei der Gewerbesteuer, die im Kern eine Gemeindesteuer darstellt, nur bezogen auf den Gesamthaushalt sinnvoll interpretiert werden ${ }^{7}$. Abb. 5 ist analog zu Abb. 4 für die Grundsteuer aufgebaut und zeigt neben den normierten auch die Grenzbelastungen zu tatsächlichen Hebesätzen.

Auch hier bewirken die Bundesergänzungszuweisungen für unterproportionale Gemeindesteuerkraft einen Anstieg der Grenzbelastungen um rund 50 Prozentpunkte. Die Empfängerländer verlieren im Gesamthaushalt $16,2 \%$ bis $20,3 \%$ ihrer Mehreinnahmen. Die aggregierten Grenzbelastungen berechnet zu tatsächlichen Hebesätzen liegen um bis zu 11,6 Prozentpunkte (SL) unter bzw. bis zu 30,5 Prozentpunkte (BB) über den Grenzbelastungen zum normierten Hebesatz.

Die Gewerbesteuer-Grenzbelastungen sind bei allen Ländern mit Ausnahme der Bezieher von Bundesergänzungszuweisungen für unterproportionale Gemeindesteuerkraft ein wenig größer als die Grundsteuer-Grenzbelastungen. Dieser Effekt basiert darauf, dass bei der Gewerbesteuer ein Teil der Mehreinnahmen im Landeshaushalt verbucht und voll als Finanzkraftzuwachs des Landes veranlagt wird. Bei der Grundsteuer werden dagegen alle Mehreinnahmen den Gemeinden zugerechnet und nur anteilig als Finanzkraft des Landes gewertet. Letzteres erhöht allerdings die Grenzbelastungen der Länder, die Zuweisungen nach der Gemeindesteuerkraft erhalten. Dort müssen sie stärkere Einbußen hinnehmen, wenn die Mehreinnahmen aus der Grundsteuer stammen.

\footnotetext{
${ }^{6}$ Die Grenzbelastungen berechnet zu tatsächlichen Hebesätzen ergeben sich aus den Grenzbelastungen zum normierten Hebesatz dividiert durch die Relation zwischen dem tatsächlichen Hebesatz $(\mathrm{H})$ und dem bundesdurchschnittlichen Hebesatz $\left(\mathrm{H}_{\varnothing}\right)$. Die Relation $\mathrm{H} / \mathrm{H}_{\varnothing}$ entspricht dem Verhältnis der tatsächlichen Einnahmen $(\mathrm{H} \times$ Grundbetrag) zu den normierten Einnahmen $(\mathrm{H} \varnothing \times$ Grundbetrag).

7 Bezogen auf den Landeshaushalt lassen sich die Grenzbelastungen zwar rechnerisch bestimmen. Infolge des sehr kleinen Länderanteils erreichen sie jedoch exorbitante Werte und sind wenig aussagefähig.
} 


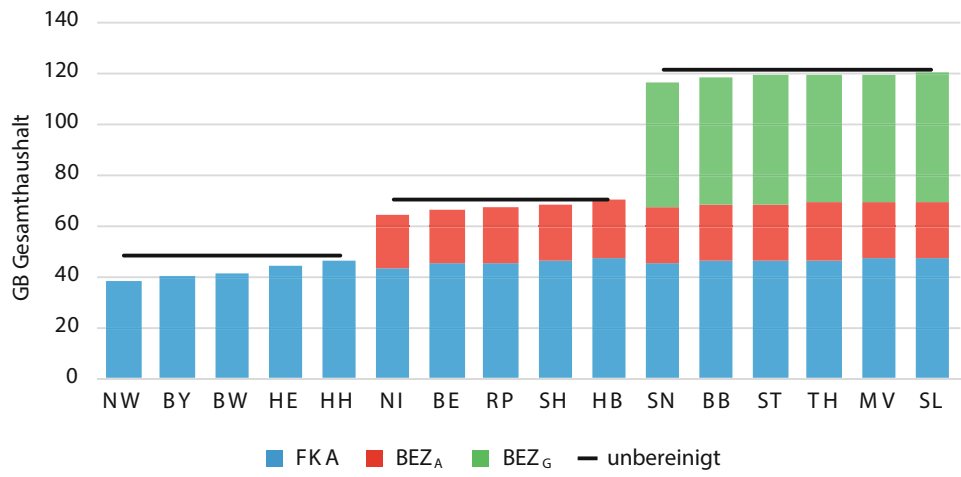

\begin{tabular}{|l|rrrrrrrrrrrrrrrr|r|}
\hline Land & $\mathrm{NW}$ & $\mathrm{BY}$ & $\mathrm{BW}$ & $\mathrm{HE}$ & $\mathrm{HH}$ & $\mathrm{NI}$ & $\mathrm{BE}$ & $\mathrm{RP}$ & $\mathrm{SH}$ & $\mathrm{HB}$ & $\mathrm{SN}$ & $\mathrm{BB}$ & $\mathrm{ST}$ & $\mathrm{TH}$ & $\mathrm{MV}$ & $\mathrm{SL}$ & $\mathrm{GB}$ \\
\hline $\mathrm{FKA}$ & 37,9 & 40,6 & 41,7 & 44,4 & 46,6 & 43,5 & 45,2 & 45,7 & 46,3 & 47,5 & 45,7 & 46,5 & 46,7 & 46,8 & 47,0 & 47,4 & 48,0 \\
$\mathrm{BEZ}_{\mathrm{A}}$ & & & & & & 20,4 & 21,2 & 21,5 & 21,8 & 22,3 & 21,5 & 21,9 & 22,0 & 22,0 & 22,1 & 22,3 & 22,5 \\
$\mathrm{BEZ}_{\mathrm{G}}$ & & & & & & & & & & & 49,1 & 49,8 & 50,0 & 50,0 & 50,3 & 50,6 & 51,1 \\
\hline$\sum \mathrm{GB}_{\mathrm{LG}}$ & 37,9 & 40,6 & 41,7 & 44,4 & 46,6 & 63,9 & 66,4 & 67,1 & 68,1 & 69,7 & 116,2 & 118,2 & 118,6 & 118,8 & 119,4 & 120,3 & 121,6 \\
\hline$\sum \mathrm{GB}_{\mathrm{H}}$ & 33,8 & 43,6 & 45,7 & 43,3 & 39,9 & 63,1 & 65,2 & 71,5 & 72,1 & 63,0 & 110,8 & 148,7 & 131,4 & 116,9 & 126,1 & 108,7 & 121,6 \\
$\Delta \mathrm{GB}$ & $-4,1$ & 2,9 & 4,0 & $-1,2$ & $-6,7$ & $-0,8$ & $-1,3$ & 4,3 & 4,0 & $-6,7$ & $-5,4$ & 30,5 & 12,8 & $-1,8$ & 6,8 & $-11,6$ & 0,0 \\
$\mathrm{H} / \mathrm{H}_{\varnothing}$ & 112,3 & 93,3 & 91,3 & 102,7 & 116,7 & 101,2 & 102,0 & 93,9 & 94,5 & 116,9 & 104,9 & 79,5 & 90,3 & 101,6 & 94,6 & 110,7 & 100,0 \\
\hline
\end{tabular}

Abb. 5 Grenzbelastungen bei der Gewerbesteuer

\section{Abbau der Grenzbelastungen}

Die Grenzbelastungen, die bei einer Erhöhung der Steuereinnahmen auftreten, ,erzeugen negative Anreizeffekte, weil Anstrengungen zur Verbesserung der eigenen Wirtschafts- und Steuerkraft sich zumindest finanziell für ein Bundesland kaum auszahlen. Dabei ist zu berücksichtigen, dass die Länder zwar die Kosten ihrer Aktivitäten tragen, aber die daraus resultierenden Steuereinnahmen zu einem großen Teil an die anderen Bundesländer und an den Bund abführen müssen" (Scherf 2020, S. 15).

Wenn der Fokus auf den Landeshaushalt gelegt wird, ergeben sich bei der Lohnsteuer besonders hohe Grenzbelastungen bis zu $135 \%$. Dieser Blickwinkel überzeichnet allerdings das Problem. Der Gesamthaushalt von Ländern und Gemeinden stellt grundsätzlich die aussagefähigere Bezugsgröße dar, weil Land und Gemeinden die öffentlichen Leistungen auf ihrem Gebiet gemeinsam erbringen. Zudem kann das Land seine Gemeinden über den kommunalen Finanzausgleich an den Mehr- oder Mindereinnahmen im Länderfinanzausgleich beteiligen.

Im Gesamthaushalt treten allerdings ebenfalls extrem hohe Grenzbelastungen auf. Bei der Lohnsteuer erreichen die effektiven Spitzenbelastungen fast $100 \%$. Bei der Abgeltungsteuer liegen sie mit $98 \%$ und bei der Körperschaftsteuer mit $92 \%$ nur wenig darunter. Die höchsten Spitzenbelastungen finden sich freilich bei den kommunalen Steuern mit $120 \%$ für die Gewerbesteuer und $122 \%$ für die Grundsteuer.

Als Triebfeder der Spitzenbelastungen erweisen sich die neuen Bundesergänzungszuweisungen für unterproportionale Gemeindesteuerkraft. Sie erhöhen die Grenzbelastungen bei der Lohnsteuer um ca. 14 und bei den Gemeindesteuern 


\begin{tabular}{|c|c|c|c|c|c|c|c|c|c|c|c|c|c|c|c|c|c|}
\hline Land & NW & BY & BW & $\mathrm{HE}$ & $\mathrm{HH}$ & $\mathrm{NI}$ & $\mathrm{BE}$ & $\mathrm{RP}$ & SN & SH & BB & ST & $\mathrm{TH}$ & MV & SL & $\mathrm{HB}$ & $\mathrm{GB}_{\mathrm{U}}$ \\
\hline & \multicolumn{17}{|c|}{ Lohnsteuer } \\
\hline$\sum \mathrm{GB}_{\mathrm{LG} 1}$ & 46,5 & 49,8 & 51,2 & 54,6 & 57,2 & 78,5 & 81,6 & 82,4 & 83,6 & 85,6 & 95,8 & 97,6 & 98,0 & 98,1 & 98,7 & 99,4 & 100,5 \\
\hline$\Delta \mathrm{GB}$ & & & & & & & & & & & $-13,4$ & $-13,6$ & $-13,7$ & $-13,7$ & $-13,7$ & $-13,8$ & $-14,0$ \\
\hline \multirow[t]{2}{*}{$\sum \mathrm{GB}_{\mathrm{LG2}}$} & 46,5 & 49,8 & 51,2 & 54,6 & 57,2 & 78,5 & 81,6 & 82,4 & 83,6 & 85,6 & 82,4 & 84,0 & 84,3 & 84,4 & 84,9 & 85,6 & 86,6 \\
\hline & \multicolumn{17}{|c|}{ Gewerbesteuer } \\
\hline$\sum \mathrm{GB}_{\mathrm{LG} 1}$ & 37,9 & 40,6 & 41,7 & 44,4 & 46,6 & 63,9 & 66,4 & 67,1 & 68,1 & 69,7 & 116,2 & 118,2 & 118,6 & 118,8 & 119,4 & 120,3 & 121,6 \\
\hline$\Delta \mathrm{GB}$ & & & & & & & & & & & $-49,1$ & $-49,8$ & $-50,0$ & $-50,0$ & $-50,3$ & $-50,6$ & $-51,1$ \\
\hline$\sum \mathrm{GB}_{\mathrm{LG} 2}$ & 37,9 & 40,6 & 41,7 & 44,4 & 46,6 & 63,9 & 66,4 & 67,1 & 68,1 & 69,7 & 67,2 & 68,4 & 68,7 & 68,7 & 69,1 & 69,7 & 70,5 \\
\hline
\end{tabular}

Abb. 6 Grenzbelastungen ohne BEZ nach der Gemeindesteuerkraft

sogar um ca. 50 Prozentpunkte. Diese speziellen Bundesergänzungszuweisungen bewirken einen Steuerkraftausgleich, der den Finanzkraftausgleich unsystematisch überlagert und damit Übernivellierungseffekte erzeugt (Scherf 2020, S. 10-12). Insofern bietet sich die Abschaffung der besonders anreizfeindlichen Bundesergänzungszuweisungen für unterproportionale Gemeindesteuerkraft als erstrangige Reformoption an.

Für die vom Steueraufkommen her wichtigsten Fälle der Lohnsteuer und der Gewerbesteuer zeigt Abb. 6 die deutliche Reduktion der Grenzbelastungen, die durch einen Wegfall der Gemeindesteuerkraftzuweisungen erreicht werden kann. Bezogen auf den Gesamthaushalt ergeben sich nun bei der Gewerbesteuer für den Länderfinanzausgleich geradezu moderate Belastungsquoten unter $70 \%$. Auch bei der Lohnsteuer ist die Entlastung unverkennbar, obwohl die Grenzbelastungen infolge der allgemeinen Bundesergänzungszuweisungen immer noch bis zu $86 \%$ erreichen.

Die Empfänger der Bundesergänzungszuweisungen für unterproportionale Gemeindesteuerkraft werden eine solche Reform dennoch nicht ohne Weiteres begrüßen. Zwar befinden sie sich derzeit in einer Armutsfalle, in der sich Anstrengungen zur Verbesserung der Wirtschafts- und Steuerkraft finanziell nicht lohnen, aber die Aussicht auf eigene Entwicklungschancen dürfte politisch weniger schwer wiegen als der unmittelbare Einnahmenverlust. Einen Ausweg aus diesem Dilemma bieten möglicherweise Pauschaltransfers, die den Einnahmenverlust zunächst voll kompensieren und in den darauf folgenden Jahren schrittweise abgebaut werden können (Scherf 2020, S. 22-25). ${ }^{8}$

\section{Zusammenfassung}

Der Beitrag präsentiert ein Modell zur Analyse der Grenzbelastungen im Länderfinanzausgleich, das mit wenigen Basisinformationen auskommt. Man muss den komplexen Länderfinanzausgleich nicht komplett simulieren, um die Grenzbelastungen transparent und differenziert für alle relevanten Steuern auszuleuchten. Als Ausgangsdaten werden nur die Einwohnerzahlen der Bundesländer und die Einwoh-

\footnotetext{
8 Durch Pauschaltransfers ließen sich auch die allgemeinen Bundesergänzungszuweisungen ersetzen und die negativen Anreizeffekte des Länderfinanzausgleichs damit noch einmal deutlich reduzieren. Allerdings dürften die politischen Widerstände gegen eine so umfassende Reform recht hoch sein.
} 
nergewichte bei den Länder- und Gemeindesteuern benötigt. Alle weiteren Schlussfolgerungen ergeben sich dann unmittelbar aus den Parametern des Systems und einigen Strukturgleichungen. Ein zentraler Vorteil des Modells besteht darin, dass die Teilursachen der Grenzbelastungen auf den verschiedenen Ausgleichsstufen separat ausgewiesen und ihre Beiträge zur Gesamtbelastung damit klar erkennbar werden.

Mithilfe des Modells lassen sich nicht nur die Grenzbelastungen des aktuellen Systems, sondern auch eine Vielzahl von Parametervariationen analysieren. Beispielsweise lässt sich leicht zeigen, dass die häufig geforderte vollständige Anrechnung der kommunalen Steuerkraft die Grenzbelastungen für sich genommen deutlich vergrößert und somit dem Ziel einer Verbesserung der Anreizeffekte entgegensteht.

Die teils enormen Grenzbelastungen, die im Länderfinanzausgleich eintreten, verdienen weit größere Aufmerksamkeit als ihnen im politischen Entscheidungsprozess eingeräumt wird. Bei der letzten Reform wurden Hinweise auf die besondere Problematik der Bundesergänzungszuweisungen für unterproportionale Gemeindesteuerkraft offenkundig ignoriert. Vor diesem Hintergrund erscheint es dringlich, die Debatte über die negativen Anreizeffekte des Länderfinanzausgleichs erneut zu intensivieren. Das heutige System, in dem sich ein Land finanziell verbessern kann, wenn seine Steuereinnahmen sinken, ist jedenfalls auf Dauer mit einer nachhaltigen föderalen Finanzordnung unvereinbar (Büttner und Görbert 2016, S. 824).

Funding Open Access funding provided by Projekt DEAL.

Open Access Dieser Artikel wird unter der Creative Commons Namensnennung 4.0 International Lizenz veröffentlicht, welche die Nutzung, Vervielfältigung, Bearbeitung, Verbreitung und Wiedergabe in jeglichem Medium und Format erlaubt, sofern Sie den/die ursprünglichen Autor(en) und die Quelle ordnungsgemäß nennen, einen Link zur Creative Commons Lizenz beifügen und angeben, ob Änderungen vorgenommen wurden.

Die in diesem Artikel enthaltenen Bilder und sonstiges Drittmaterial unterliegen ebenfalls der genannten Creative Commons Lizenz, sofern sich aus der Abbildungslegende nichts anderes ergibt. Sofern das betreffende Material nicht unter der genannten Creative Commons Lizenz steht und die betreffende Handlung nicht nach gesetzlichen Vorschriften erlaubt ist, ist für die oben aufgeführten Weiterverwendungen des Materials die Einwilligung des jeweiligen Rechteinhabers einzuholen.

Weitere Details zur Lizenz entnehmen Sie bitte der Lizenzinformation auf http://creativecommons.org/ licenses/by/4.0/deed.de.

\section{Literatur}

Bundesministerium der Finanzen (2020). Erste Verordnung zur Durchführung des Finanzausgleichsgesetzes im Ausgleichsjahr 2020. Bundesrat Drucksache 62/20. Berlin: Bundesministerium der Finanzen.

Burret, H.T., Bury, Y., \& Feld, L. (2018). Grenzabschöpfungsraten im deutschen Finanzausgleich. List Forum, 44, 1-22.

Büttner, T., \& Görbert, T. (2016). Neuregelung des bundesstaatlichen Finanzausgleichs. Wirtschaftsdienst, $11,818-824$.

Scherf, W. (2020). Länderfinanzausgleich 2020: Neue Form - alte Probleme. Finanzwissenschaftliche Arbeitspapiere, Bd. 102/2020. Gießen: Justus-Liebig-Universität.

Statistisches Bundesamt (2019). Realsteuervergleich. Fachserie 14 Reihe 10.1. Wiesbaden: DeStatis. 